\title{
Asociación entre postura cráneo cervical y maloclusiones Una revisión
}

\author{
Association between craneocervical posture and maloclusions. A review \\ Mariela Ramírez-Velásquez 이 ${ }^{1,2 a} ;$ Eduary Rodulfo $^{(0)}$; Cristián Urgilés ${ }^{1 \mathrm{c}}{ }^{\text {; }}$; Karina Herrera-Espinoza \\ 1d. Denisse Ludizaca-Llerena ${ }^{1 \mathrm{~d}}$
}

\begin{abstract}
Resumen
La relación biomecánica entre el segmento cráneo cervical y las alteraciones de forma y función del sistema estomatognático ha sido un tema muy controversial, por su influencia en numerosos y distintos aspectos con los cuales se le vincula, como la función respiratoria, disfunciones de la articulación temporomandibular, o relaciones maxilomandibulares. El objetivo de este estudio fue determinar la asociación entre las alteraciones de la postura cráneo cervical y las maloclusiones, mediante una revisión utilizando como estrategias, la búsqueda en las bases de datos PubMed (Medline), SciELO; Redalyc, LILACS; se incluyeron articulos comprendidos entre 2015-2019 publicados en idiomas ingles, español y/o portugues. Se obtuvieron 445 publicaciones, 427 fueron excluidas luego de la evaluación de los títulos y resúmenes, quedando 18 artículos para la lectura y análisis, resultando finalmente 8 artículos incluidos en este estudio. Se determinó que existe poca evidencia científica con diseños apropiados para determinar una asociación entre la relación biomecánica de cabeza, columna cervical y piezas dentarias, además se evidencia la falta de homogeneidad entre las mediciones que determinan las alteraciones del segmento cráneo cervical en los distintos estudios.
\end{abstract}

Palabras clave: Maloclusión; Postura cervical; Postura cráneo cervical. (Fuente: DeCS BIREME)

\section{Abstract}

The biomechanical relationship between the cranio-cervical segment and alterations in the form and function of the stomatognathic system has been a highly controversial topic, due to its influence on numerous and different aspects with which it is linked, such as respiratory function, temporomandibular joint dysfunctions, or maxillomandibula relationships. The objective of this study was to determine the association between the alterations of the cervical cranial posture and the malocclusions, by means of a bibliographic review using as strategies, the search in the databases PubMed (Medline), SciELO; Redalyc, LILACS; articles between 2015-2019 published in English, Spanish and/or Portuguese were included. A total of 445 publications were obtained, 427 were excluded after the evaluation of titles and abstracts, leaving 18 articles for reading and analysis, finally resulting in 8 articles included in this study. It was determined that there is little scientific evidence with appropriate designs to determine an association between the biomechanical relationship of the head, cervical spine and teeth, in addition there is evidence of lack of homogeneity between the measurements that determine the alterations of the cranio-cervical segment in the different studies.

Keywords: Malocclusion; Cervical posture; Craneocervical posture. (Source: MeSH NLM)

\footnotetext{
${ }^{1}$. Universidad Católica de Cuenca (UCACUE) sede Azogues. Carrera Odontología. Ecuador.

2. Centro de Investigación UCACUE sede Azogues. Ecuador.

${ }^{a}$ Docente investigadora. Coordinadora del Centro de Investigación.

${ }^{6}$ Especialista en Ortopedia Maxilar

'Docente

¿.Estudiante

Correspondencia:

Mariela Ramírez

Correo electrónico: mramirezv@ucacue.edu.ec

Dirección: Universidad Católica de Cuenca

Av. 16 de abril y Ernesto Che Guevara. Azogues, Ecuador, 030102
}

Este es un artículo de acceso abierto distribuido bajo la licencia Creative Commons Atribución 4.0 Internacional (CC BY 4.0) https://creativecommons.org/licenses/by/4.0/deed.es

Citar como: Ramírez-Velásquez M, Rodulfo E, Urgilés C, Herrera-Espinoza K, Ludizaca-Llerena D. Asociación entre postura cráneo cervical y maloclusiones. Una revisión. KIRU. 2021; 18(1): 55-64. https://doi.org/10.24265/kiru.2021.v18n1.8 


\section{INTRODUCCION}

La salud bucal es definida como la ausencia de múltiples enfermedades que pueden llegar a afectar la salud del sistema estomatognático (SE). Una maloclusión o una mala relación de los maxilares puede estar asociado a hábitos deletéreos ${ }^{(1-3)}$ e influenciar la estética dental ${ }^{(4)}$ y facial ${ }^{(5-8)}$. Varios estudios (9-11) muestran la alta prevalencia de maloclusiones en distintos países, así mismo lo evidencia un estudio de revisión sistemática ${ }^{(12)}$ que tuvo por objetivo agrupar datos para determinar la distribución de los rasgos de mal oclusiones a nivel mundial en denticiones mixtas $y$ permanentes, indicando que, en estas denticiones, la maloclusión clase I es más frecuente que la clase II, específicamente entre los africanos; y la menos frecuente la clase III.

Hoy en día se considera al SE como parte de la unidad cráneo cérvico mandibular donde los componentes que la constituyen guardan estrecha relación y donde la posición ortostática de la cabeza sobre la columna cervical influencia dicha unidad ${ }^{(13)}$.

Diferentes investigaciones en la actualidad tratan de relacionar las discrepancias sagitales y verticales con la postura de la cabeza (PC) (14-21), incluso se le ha relacionado con los desórdenes temporomandibulares ${ }^{(22-26)}$.

La postura cráneo cervical (PCC) y mandibular están condicionadas al equilibrio neuromuscular que debe existir entre ellas por lo cual diversos factores pueden influir sobre la postura de ambas, como lo son los hábitos orales ${ }^{(27-29)}$ debido a la relación anatómica y funcional de las diversas estructuras dentofaciales y de la columna cervical que pudieran modificar la posición postural existente.

Existe evidencia que afirman una asociación estadísticamente débil entre alteraciones de la postura cráneo cervical y las maloclusiones, como por ejemplo los resultados de Aldana ${ }^{(30)}$ cuya investigación tuvo como objetivo contrastar la hipótesis nula que indicaba que "no hay asociación entre maloclusiones y alteraciones posturales de cabeza y cuello" en este estudio descriptivo transversal en pacientes que consultaron por tratamiento de ortodoncia, cuya muestra estuvo constituida por 116 pacientes con maloclusión, se buscó asociación entre los valores del análisis cráneo cervical de Rocabado, el cefalograma, estudio de modelos y la evaluación funcional de la lengua resultando asociaciones estadísticas débiles entre la rotación anti horaria del cráneo (ángulo cráneo vertebral y distancia intervertebral C0C1 disminuidas), con clase III esqueletal que además tenían rotación posterior mandibular y que eran dólico faciales. Altos valores del triángulo hioideo, se asociaron también débilmente, con Clase II esqueletal, junto con alteraciones de la posición de la lengua en reposo y retrusión dentaria.

En espera de orientar e incentivar investigaciones relacionadas al tema $y$ debido a las tantas controversias sobre el mismo, esta revisión tiene como objetivo determinar la asociación de las alteraciones de la PCC y las maloclusiones mediante la definición de la literatura existentedurante el periodo 2015-2019.

\section{MATERIAL Y MÉTODOS}

Se realizó una revisión no sistemática, para responder a la siguiente pregunta de investigación: ¿Existe asociación entre las alteraciones de la PCC y las maloclusiones?

La estrategia de búsqueda incluyó las bases de datos científicas PubMed (Medline), SciELO; Redalyc; LILACS. Se realizó una búsqueda electrónica de las publicaciones utilizando las palabras clave: "craniocervical OR postural AND malocclusion", "malocclusion AND posture", "craniocervical OR posture AND stomatognathic system", "head extended AND posture", "head extended AND distooclusion", "head AND, "distooclusion AND posture", "craniocervical AND distooclusion", "AND head extended". $\mathrm{Se}$ incluyeron estudios transversales, de cohorte y estudios de casos y controles, del periodo comprendido entre 2019-2015 publicados en el idioma ingles, español y portugués, con alteraciones sagitales y postura craniocervical como variables de estudio, sin restricción de edades en los sujetos. Se excluyeron artículos de revision y los que no abordaran especificamente el tema. Se obtuvieron títulos y resúmenes, antecedentes $\mathrm{y}$ objetivos, métodos $\mathrm{y}$ resultados.

Se evalúo la calidad de los artículos incluidos a partir de la declaración CONSORT 2010, por parte de 3 revisores (MR, ER, CU), incluyendo estudios que se encontraron por encima del $80 \%$ en la calidad del estudio. 


\section{RESULTADOS}

De la búsqueda electrónica se obtuvieron un total de 445 artículos, 144 fueron ubicados en PubMed, 62 artículos en SciELO, 33 en LILACS: y 206 de REDALYC. 427 artículos se excluyeron luego de la evaluación de título y resumen por no estar claramente relacionados al tema de investigación, quedando 18 para la lectura completa del mismo, de manera independiente por los revisores. Un total de 8 artículos se incluyen en este estudio. (Figura 1)

Asociación de las alteraciones de la PCC y las maloclusiones.
En la Tabla 1 se muestran los resultados de 5 artículos $(15,18,19,20,31)$ que evidencian asociación entre PCC y las maloclusiones.

En la tabla 2 se indica la asociación encontrada por los autores entre las variables estudiadas.

\section{No asociación de las alteraciones de la PCC y las maloclusiones}

La Tabla 3 muestra los estudios que no encontraron asociación entre PCC y maloclusiones, donde se incluyeron tres estudios $(14,17,22)$.

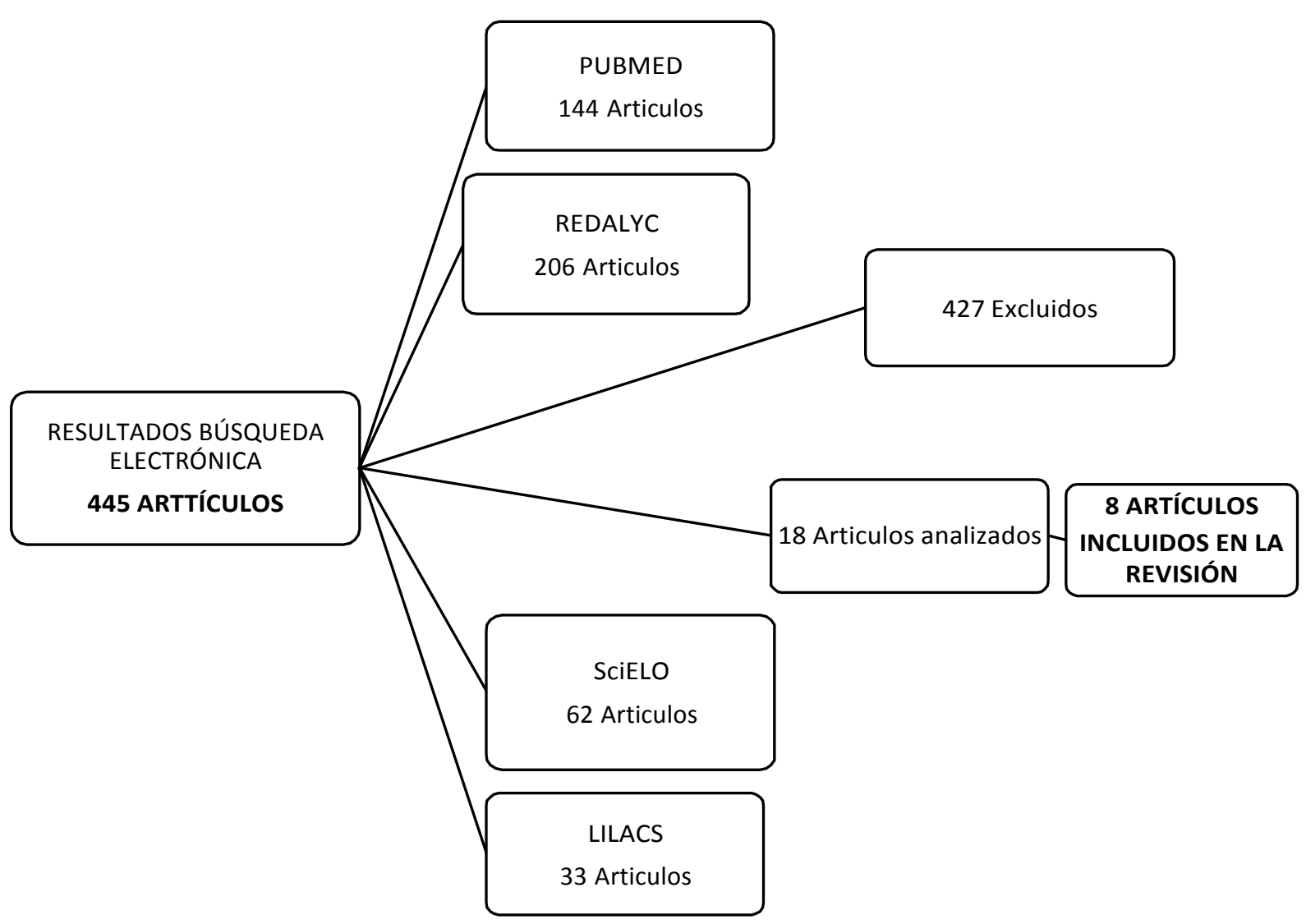

Figura 1. Resultados de la búsqueda electrónica 
Tabla 1. Artículos que evidencian la asociación entre postura cráneo cervical y maloclusiones.

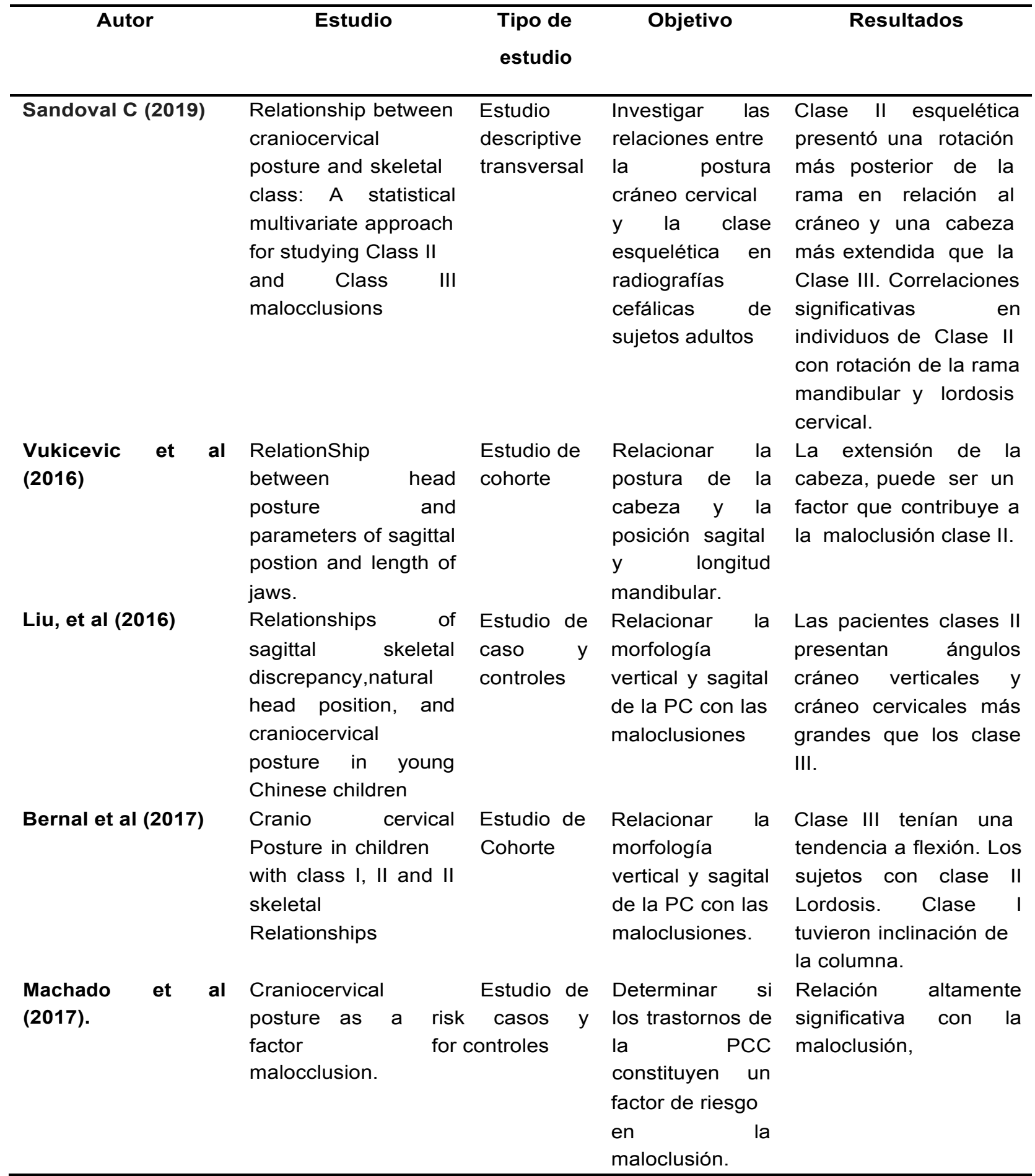


Tabla 2. Asociación entre alteraciones de postura cráneo cervical y maloclusiones

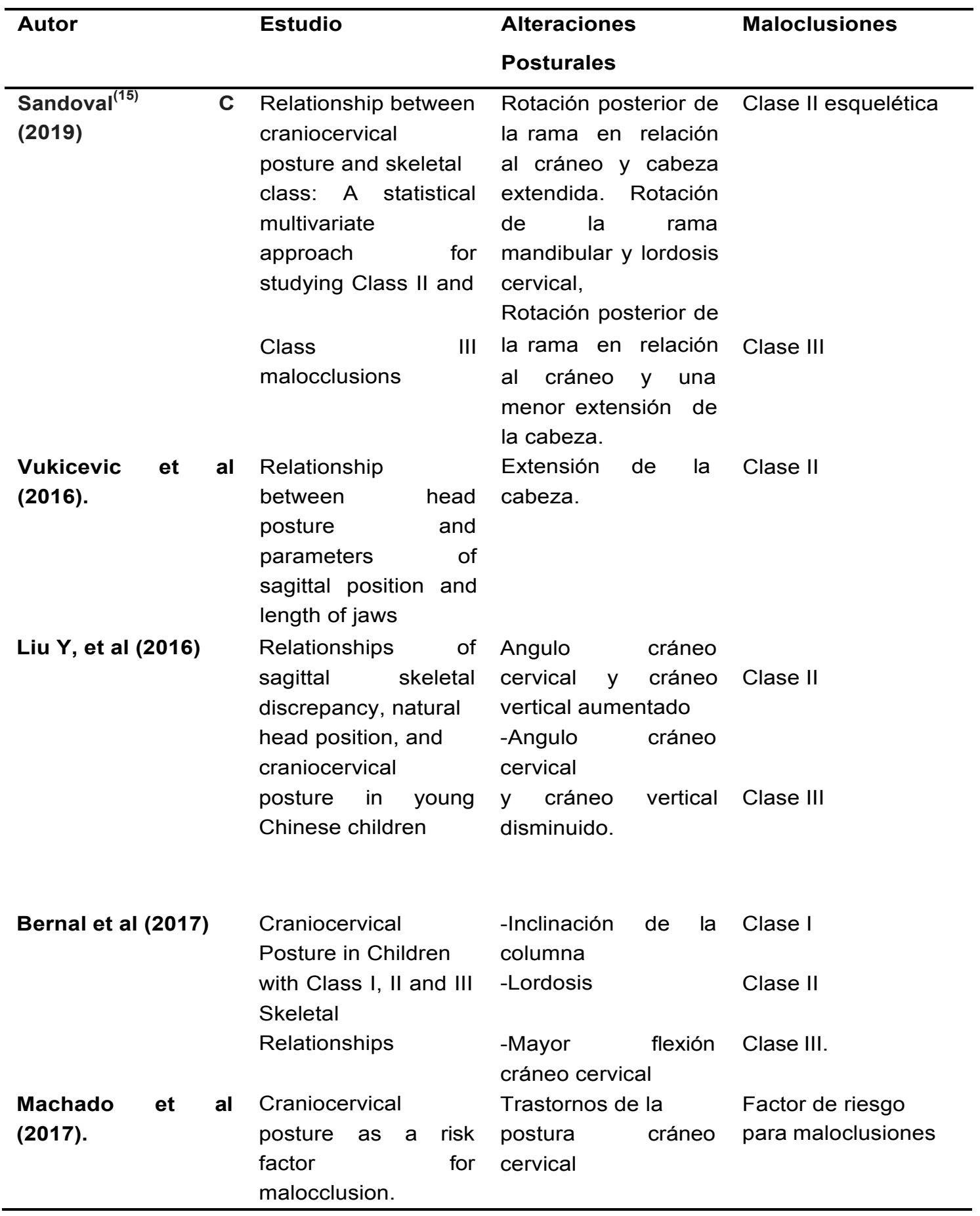


Tabla 3: Estudios que no evidencian asociación entre postura cráneo cervical y maloclusiones.

\begin{tabular}{|c|c|c|c|c|}
\hline Autor & Estudio & $\begin{array}{ll}\text { Tipo de } \\
\text { Estudio }\end{array}$ & Objetivo & Resultados \\
\hline $\begin{array}{c}\text { González } \\
\text { Rodríguez }\end{array}$ & $\begin{array}{l}\text { Relación entre oclusión } \\
\text { dentaria y postura } \\
\text { cráneo-cervical en } \\
\text { niños con } \\
\text { maloclusiones clase II } \\
\text { y III. }\end{array}$ & $\begin{array}{l}\text { descriptivo } \\
\text { transversal }\end{array}$ & $\begin{array}{l}\text { Describir la relación entre } \\
\text { la oclusión dentaria y la } \\
\text { postura del sistema } \\
\text { cráneo-cervical en niños } \\
\text { con maloclusiones clase } \\
\text { II y clase III }\end{array}$ & $\begin{array}{ll}\text { Posición de la } \\
\text { cabeza } & \text { normal } \\
\text { para clase I y II }\end{array}$ \\
\hline $\begin{array}{l}\text { Di } \\
\text { Giacomo } \\
\text { et al } \\
(2018)\end{array}$ & $\begin{array}{l}\text { Relationship between } \\
\text { cervical spine and } \\
\text { skeletal class II in } \\
\text { subjects with and } \\
\text { without } \\
\text { temporomandibular } \\
\text { disorders }\end{array}$ & $\begin{array}{lr}\text { Estudio de } \\
\text { casos y } \\
\text { controles }\end{array}$ & $\begin{array}{l}\text { Evaluar los cambios en la } \\
\text { estructura cráneo cervical } \\
\text { y en la posición del } \\
\text { hueso hioides en sujetos } \\
\text { esqueléticos de clase II } \\
\text { con y sin trastornos } \\
\text { temporomandibulares }\end{array}$ & $\begin{array}{l}\text { No muestra } \\
\text { evidencias } \\
\text { significativas en } \\
\text { sus resultados de } \\
\text { la relación entre la } \\
\text { clase Il esquelética } \\
\text { y la columna } \\
\text { cervical. }\end{array}$ \\
\hline $\begin{array}{c}\text { Smailiené } \\
\text { et al } \\
(2017)\end{array}$ & $\begin{array}{l}\text { Effect of Treatment } \\
\text { with Twin-Block } \\
\text { Appliances on Body } \\
\text { Posture in Class II } \\
\text { Malocclusion Subjects: } \\
\text { A Prospective Clinical } \\
\text { Study }\end{array}$ & $\begin{array}{l}\text { Estudio } \\
\text { Clinico } \\
\text { Prospectivo }\end{array}$ & $\begin{array}{l}\text { Analizar el efecto del } \\
\text { tratamiento de ortodoncia } \\
\text { con un dispositivo twin- } \\
\text { block en la postura del } \\
\text { cuerpo. }\end{array}$ & $\begin{array}{l}\text { Los cambios en la } \\
\text { postura del cuerpo } \\
\text { durante el } \\
\text { tratamiento con el } \\
\text { dispositivo Twin- } \\
\text { block fueron una } \\
\text { expresión en el } \\
\text { crecimiento } \\
\text { fisiológico, no una } \\
\text { respuesta a la } \\
\text { mejora en la } \\
\text { oclusión. }\end{array}$ \\
\hline
\end{tabular}

\section{DISCUSIÓN}

La asociación entre la postura cráneo cervical y las maloclusiones, siempre es un tema controversial, por lo cual es necesario su actualización constante, pues siguen existiendo estudios que confirman o niegan tal relación. Así mismo, el análisis crítico de revisiones sistemáticas, refiere la necesidad de nuevos estudios, por presentarse problemas metodológicos en las investigaciones realizadas $y / o$ la necesidad de estudios de casos y controles o de cohortes que permitan determinar o no, la relación de las variables ${ }^{(32-34)}$. De allí que esta investigación tuvo como propósito, una revisión de la literatura para definir la información existente y determinar si existe, o no, una asociación entre la PCC y las maloclusiones.

Una de las investigaciones que correlaciona la PCC y las maloclusiones fue el estudio de Sandoval ${ }^{(15)}$ realizado en sujetos adultos, a través de sesenta y cinco radiografías cefálicas de pacientes diagnosticados como clase II y III esquelética, la postura cráneo cervical fue evaluada a través de las variables propuestas por Solow y Rocabado. Los resultados de Sandoval evidencian que pacientes diagnosticados clase II esquelética presentaron una rotación más posterior de la rama en relación al cráneo y 
una cabeza más extendida que la Clase III esquelética. Observó además correlaciones significativas en los individuos de clase II entre la rotación de la rama mandibular y la lordosis cervical, y entre, la rotación de la rama mandibular y la postura cráneo cervical.

Por su parte el estudio de Vukicevic et al (20), incluyó 90 sujetos (30 para cada clase esquelética I, II y III) cuyas edades oscilaban entre 8 y 14 años. A cada uno se le realizó el cefalograma lateral y el análisis mediante el programa inf "Onyx Ceph", El objetivo de ese estudio fue examinar la relación entre la $\mathrm{PC}$ y los parámetros de la posición sagital, así como la longitud mandibular. Se demostró que el aumento de la extensión de la cabeza en relación con la columna cervical puede ser un factor que contribuye a la formación de una maloclusión clase II, concordando con los resultados de Sandoval ${ }^{(15)}$ y Liu Y et al. ${ }^{(19)}$.

Por su parte Liu $\mathrm{Y}$ et al. (19) demostró además que los pacientes con alteraciones cráneo cervicales presentan mayor incidencia de maloclusiones clase III, extensión de la cabeza y un ángulo cráneo vertical aumentado. Este estudio tuvo como objetivo investigar las relaciones de discrepancia esquelética sagital, posición natural de la cabeza (PNC) y PCC en 90 niños chinos. Estos presentaban un tipo facial vertical, ángulo del plano mandibular de Frankfort promedio, y relaciones esqueléticas clase I, II y III según su ángulo ANB. Se tomaron radiografías cefálicas en PNC. Se midieron y compararon las variables que representan la morfología cráneo facial sagital y vertical, la postura de la cabeza y la postura cráneo cervical. Se evidenció que las pacientes clase II presentaban los ángulos cráneo vertical y cráneo cervical mayores que los pacientes clase III.

A diferencia de los estudios anteriores de, Sandoval ${ }^{(15)}$ Liu et al. ${ }^{(19)}$ y Vukicevic et al. (20), la investigación de Bernal et al (31) evidenció que los niños de clase III estudiados tenían una

tendencia a flexión cráneo cervical superior. Este estudio descriptivo de corte transversal incluyó a 107 niños, (55 niñas 52 niños), con edades entre 6 y 11 años. Niños tratados desde el punto de vista ortodóntico, ortopédico y sistémicamente sanos. Después de la debida calibración, fueron tomadas radiografías laterales de cráneo, para el diagnóstico del tratamiento ortopédico, obtenidas por el mismo operador en PNC. El análisis radiográfico se realizó con el software NEMOTEC: se midieron 13 variables registrando: edad, sexo, ángulo ANB (para clasificar las relaciones esqueléticas sagitales) y 10 variables relacionadas con la PCC.

El estudio de Machado (18) tuvo como objetivo determinar si los trastornos de la postura cráneo cervical constituían un factor de riesgo en la maloclusión, para ello se formaron 2 grupos de pacientes con y sin maloclusiones, cada uno con 90 pacientes. Para determinar la prevalencia de los trastornos de la PCC, emplearon la prueba de convergencia ocular, la prueba de rotación de la cabeza y la alteración del plano biclavicular. En el grupo con maloclusión predominaron las mujeres $(55,55 \%)$; La prevalencia de los trastornos posturales fue del $97,77 \%$ en el grupo con maloclusión contra el 48,88 \% del grupo control; obteniendo una relación muy altamente significativa entre las variables estudiadas, determinando que la postura cráneo cervical constituye un factor de riesgo que incrementa 46 veces más el riesgo de padecer maloclusiones.

Por otra parte, estudios como el de González (14) y Smailiené (17) no lograron resultados estadísticamente significativos para demostrar una correlación entre las variables estudiadas. González, tras un estudio descriptivo transversal en 19 niños, de 7 a 12 años de edad, con maloclusiones clase II y III de Angle y realizado a través de examen bucal y telerradiografía, presentó resultados predominantes de clase II, ángulo cráneo-vertebral y espacio suboccipital dentro del rango normal.

El estudio de Smailiené consistió en la evaluación de 23 niños de edad promedio de 12-16 años, los cuales se examinaron ortopédicamente (análisis forma de la espalda) y ortodoncicamente (análisis cefalométrico) antes del tratamiento con un dispositivo Twin-block y 14 meses después del tratamiento. Sus resultados evidenciaron que los cambios en la postura corporal durante el tratamiento con el 
aparato Twin-block fueron una expresión del crecimiento fisiológico, y no una respuesta a la mejora en la oclusión.

La investigación de Di Giacomo ${ }^{(22)}$, estudió la relación entre la columna cervical y la clase II esquelética en sujetos, con y sin trastornos temporomandibulares (TTM), evaluando y comparando los análisis cefalométricos de 59 sujetos con clase II esquelética. Concluyeron que la relación significativa entre ésta y la columna cervical no pudo ser destacada. Las mediciones consideradas fueron ANB como un parámetro de la clase II y la distancia CO$\mathrm{C} 1$, la distancia $\mathrm{C} 1-\mathrm{C} 2$, el ángulo cráneo cervical y la posición del hueso hioides para el análisis de la columna cervical.

\section{CONCLUSIONES}

Existe poca evidencia científica con diseños apropiados, como estudios de casos y controles y/o estudios de cohorte, para determinar una asociación entre las alteraciones de la postura cráneo cervical y las maloclusiones. Además, se evidencia la falta de homogeneidad entre las mediciones que determinan las alteraciones de la postura cráneo cervical, en los distintos estudios.

Contribuciones de autoría: MRV, ER, CU, $\mathrm{KH}$, DL diseñaron el estudio, recopilaron información, redactaron y aprobaron el artículo. MRV, ER, CU analizaron la calidad de los artículos

Fuente de financiamiento: Financiado por los autores.

Conflicto de intereses: Los autores declararon no tener conflictos de interés en este manuscrito.

\section{REFERENCIAS BIBLIOGRÁFICAS}

1. Grippaudo C, Paolantonio EG, Antonini G, Saulle R, La Torre G, Deli R. Association between oral habits, mouth breathing and malocclusion. Associazione fra abitudini viziate, respirazione orale e malocclusione. Acta Otorhinolaryngol Ital. 2016;36(5):386394. https://www.ncbi.nlm.nih.gov/pmc /articles/PMC5225794/
2. Arocha AA, Aranda GMS, Pérez PY, Granados HAE. Maloclusiones y hábitos bucales deformantes en escolares con dentición mixta temprana. MEDISAN 2016;20(4):426432 https://www.medigraphic.com/pdfs/med isan/mds-2016/mds164b.pdf.

3. Paolantonio EG, Ludovici N, Saccomanno S, La Torre G, Grippaudo C. Association between oral habits, mouth breathing and malocclusion in Italian preschoolers. Eur $J$ Paediatr Dent. $\quad$ 2019:20(3):204-208. http://ejpd.eu/EJPD_2019_20_3_7.pdf.

4. Kaieda AK, Bulgareli JV, Cunha IPD, et al. Malocclusion and dental appearance in underprivileged Brazilian adolescents. Braz Oral Res. 2019;33:e014. http://www.scielo.br/scielo.php?script=s ci arttext\&pid $=$ S1806$83242019000100211 \&$ Ing=en\&nrm=iso \&tlng=en.

5. Zouloumi ME, Tsiouli K, Psomiadis S, Kolokitha OE, Topouzelis N, Gkantidis $\mathrm{N}$. Facial esthetic outcome of functional followed by fixed orthodontic treatment of class II division 1 patients. Prog Orthod. 2019;20(1):42 https://www.ncbi.nlm.nih.gov/pmc/articl es/PMC6875534/

6. Dos Santos PR, Meneghim MC, Ambrosano GM, Filho MV, Vedovello SA. Influence of quality of life, selfperception, and self-esteem on orthodontic treatment need. Am J Orthod Dentofacial Orthop. 2017;151(1):143-147.

https://www.ajodo.org/article/S08895406(16)30574-1/pdf.

7. Calderón Montalvo, G., \& Dueñas, C. (2015). Influencia de seis maloclusiones en la percepción de inteligencia, atractivo físico y habilidades interpersonales. Odontolnvestigación, 1 (2).

https://revistas.usfq.edu.ec/index.php/o dontoinvestigacion/article/view/190/191

8. Corte CC, Silveira BL, Marquezan M. Influence of occlusal plane inclination and mandibular deviation on esthetics. Dental Press J Orthod. 2015;20(5):50-57.

https://www.ncbi.nlm.nih.gov/pmc/articl es/PMC4644919/

9. Asiry MA, AIShahrani I. Prevalence of malocclusion among school children of Southern Saudi Arabia. J Orthod Sci. 2019;8:2. https://www.ncbi.nlm.nih.gov/pmc/articl es/PMC6447337/ 
10. Traebert E , LGT Martins, KCR Pereira, SXS Costa. Malocclusion in Brazilian Schoolchildren: High Prevalence and Low Impact. 2018. riuni.unisul.br 2018;16 (2)2:163-167 https://www.riuni.unisul.br/handle/1234 5/7939.

11. Zhou X, Zhang $\mathrm{Y}$, Wang $\mathrm{Y}$, Zhang $\mathrm{H}$, Chen L, Liu $Y$. Prevalence of Malocclusion in 3- to 5-Year-Old Children in Shanghai, China. Int J Environ Res Public Health. 2017;14(3):328. https://www.ncbi.nlm.nih.gov/pmc/articl es/PMC5369163/

12. Alhammadi MS, Halboub E, Fayed MS, Labib A, El-Saaidi C. Global distribution of malocclusion traits: A systematic review. Dental Press J Orthod. 2018;23(6):40.e1-40.e10. https://www.ncbi.nlm.nih.gov/pmc/articl es/PMC6340198/

13. Rocabado M. Biomechanical relationship of the cranial, cervical, and hyoid regions. $J$ Craniomandibular Pract. 1983;1(3):61-66. https://www.tandfonline.com/doi/abs/10 .1080/07345410.1983.11677834

14. González Rodríguez $S$, Llanes Rodríguez M, Batista González N, Pedroso Ramos L, Pérez Valerino M. Relación entre oclusión dentaria y postura cráneo-cervical en niños con maloclusiones clase II y III. Rev.Med.Electrón. 2019; 41(1): 63-77. https://www.medigraphic.com/pdfs/rev medele/me-2019/me191g.pdf

15. Sandoval C, Díaz A, Manríquez G. Relationship between craniocervical posture and skeletal class: A statistical multivariate approach for studying Class II and Class III malocclusions . Cranio. 2019;1-8. https://pubmed.ncbi.nlm.nih.gov/31035 911/

16. Liu Y, Wang S, Wang C, Liu C. Relationships of vertical facial pattern, natural head position and craniocervical posture in young Chinese children. Cranio. 2018;36(5):311-317. https://pubmed.ncbi.nlm.nih.gov/28669 326/

17. Smailiené D, Intienè A, Dobradziejutė I, Kušleika G. Effect of Treatment with Twin-Block Appliances on Body Posture in Class II Malocclusion Subjects: A Prospective Clinical Study. Med Sci Monit. 2017;23:343352.

https://www.ncbi.nlm.nih.gov/pmc/articl es/PMC5279871/

18. Machado Martínez M, Cabrera García K, Martínez Bermúdez G. Postura craneocervical como factor de riesgo en la maloclusión. Rev Cubana Estomatol. 2017; 54(1):24-33. http://www.revestomatologia.sld.cu/inde X.php/est/article/view/669/616

19. Liu Y, Sun X, Chen Y, Hu M, Hou X, Liu C. Relationships of sagittal skeletal discrepancy, natural head position, and craniocervical posture in young Chinese children. Cranio.

2016;34(3):155-162.

https://www.ncbi.nlm.nih.gov/pubmed/2 6039882

20. Vukicevic V, Petrovic D. Relationship between head posture and parameters of sagittal position and length of jaws. Med Pregl. 2016;69(9-10):288-293. https://www.ncbi.nlm.nih.gov/pubmed/2 9693851

21. Cárdenas J M, Flores J C, Gutiérrez Cantú $\mathrm{F}$ J, Cárdenas Gylmar M, Sánchez Meraz W, Guerrero Barrera A L. Estudio Morfométrico de la Posición Cráneo-Cervical en Pacientes con Clases Esqueletales II y III. Int. J. Morphol. 2015 Jun ; 33( 2 ): 415419. https://scielo.conicyt.cl/pdf/ijmor phol/v33n2/art01.pdf

22. Di Giacomo P, Ferrara V, Accivile E Ferrato G, Polimeni A, Di Paolo C. Relationship between Cervical Spine and Skeletal Class II in Subjects with and without Temporomandibular Disorders. Pain Res Manag. 2018;2018:4286796.

http://downloads.hindawi.com/journals/ prm/2018/4286796.pdf

23. Espinosa De Santillana, I. (2018). Alteraciones posturales frecuentes en pacientes con diferentes tipos de trastornos temporomandibulares. Rev. salud pública. 2018;20(3):384-389. https://revistas.unal.edu.co/index.php/r evsaludpublica/article/view/53529

24. Cortese S, Mondello A, Galarza R, Biondi A. Postural alterations as a risk factor for temporomandibular disorders. Alteraciones posturales como factor de riesgo para trastornos témporomandibulares. Acta Odontol Latinoam. 2017;30(2):57-61. http://www.scielo.org.ar/pdf/aol/v30n2/v 30n2a02.pdf

25. Saddu SC, Dyasanoor S, Valappila NJ, Ravi BV. The Evaluation of Head and Craniocervical Posture among Patients with and without Temporomandibular Joint Disorders- A Comparative Study. $J$ Clin Diagn Res. 2015;9(8). https://www.ncbi.nlm.nih.gov/pmc/articl es/PMC4576642/

26. An JS, Jeon DM, Jung WS, Yang IH, Lim WH, Ahn SJ. Influence of temporomandibular joint disc displacement on craniocervical posture and hyoid bone position. Am J Orthod Dentofacial Orthop. 2015;147(1):72-79. https://pubmed.ncbi.nlm.nih.gov/25533 074/ 
27. Moya María P, Olate Sergio, Baeza Juan P. Análisis Craneocervical en Sujetos con Respiración Oral y Nasal. Int. J. Morphol. 2019; 37( 2 ): 724-729 https://scielo.conicyt.cl/scielo.php?scrip $\mathrm{t}=$ sci abstract\&pid=S071795022019000200724\&Ing=en\&nrm=iso \&tIng $=\mathrm{es}$

28. Milanesi Jovana M., Berwig Luana C., Busanello-Stella Angela R., Trevisan Maria Elaine, Silva Ana Maria T. da, Corrêa Eliane C. R.. Nasal patency and craniocervical posture in scholar children. Fisioter. Pesqui. [Internet]. 2017 ; 24 ( 3 ): 327-333.

http://www.scielo.br/scielo.php?script=s ci arttext\&pid=S180929502017000300327

29. Šidlauskienè $M$, Smailienè $D$, Lopatienè K, Čekanauskas E, Pribuišienè R, Šidlauskas M. Relationships Between Malocclusion, Body Posture, and Nasopharyngeal Pathology in Pre-Orthodontic Children. Med Sci Monit . 2015; 21: 1765-1773. https://www.ncbi.nlm.nih.gov/pmc/articl es/PMC4484615/

30. Aldana $P$ Alejandra, Báez $R$ Jéssica, Sandoval C Carolina, Vergara N Cristian, Cauvi L Doris, Fernández de la Reguera Alejandro. Asociación entre Maloclusiones y Posición de la Cabeza y Cuello. Int. J. Odontostomat. [Internet]. 2011 Ago [citado 2020 Nov 18] ; 5( 2 ): 119125. Disponible en: https://scielo.conicyt.cl/scielo.php?scrip $\mathrm{t}=$ sci_arttext\&pid=S0718-

381X2011000200002\&lng=es. http://dx .doi.org/10.4067/S0718-

381X2011000200002.

31. Bernal L; Marin, H; Herrera C ; Montoya C; Herrera Y. Craniocervical Posture in Children with Class I, II and III Skeletal Relationships Pesqui. bras. odontopediatria clín. integr ; 17(1): e3038, 13/01/2017 http://revista.uepb.edu.br/index.php/pbo ci/article/view/3038/pdf

32. Julià-Sánchez S, Álvarez-Herms J, Burtscher M. Dental occlusion and body balance: A question of environmental constraints?. J Oral Rehabil. 2019;46(4):388-397. https://pubmed.ncbi.nlm.nih.gov/30664 820/

33. Ramirez M., Rodulfo E., Zambrano., Rivera L. Alteración de la postura craneocervical y su asociación con las alteraciones del Sistema estomatognático. Revisión sistemática. Ciencia odontológica. 2018; 15(1):4755.

https://produccioncientificaluz.org/index .php/cienciao/article/view/24610/25055

34. González Rodríguez Suami, Llanes Rodríguez Maiyelin, Pedroso Ramos Lucía. Modificaciones de la oclusión dentaria y su relación con la postura corporal en Ortodoncia. Revisión bibliográfica. Rev haban cienc méd [Internet]. 2017 Jun [citado 2020 Mar 20] ; 16( 3 ): 371386.

Mariela Ramírez-Velásquez

ORCID iD: 난 http://orcid.org/0000-0001-7041-4346

mramirezv@ucacue.edu.ec

Eduary Rodulfo

ORCID iD: (1) http://orcid.org/0000-0001-5658-9908

rodulfoeduary@yahoo.com

Christian Urgiles

ORCID iD: (1) http://orcid.org/0000-0003-4077-4601

curgilesu@ucacue.edu.ec

Karina Herrera-Espinoza

ORCID iD: (1) http://orcid.org/0000-0001-8608-9672

klherrerae46@est.ucacue.edu.ec

Denisse Ludizaca-Llerena

ORCID iD: (1) http://orcid.org/0000-0001-9754-5936

daludizacal37@est.ucacue.edu.ec

Copyright $($ La revista. La revista Kiru es publicada por la Facultad de Odontología de la Universidad de San Martín de Porres, en Lima, Perú 